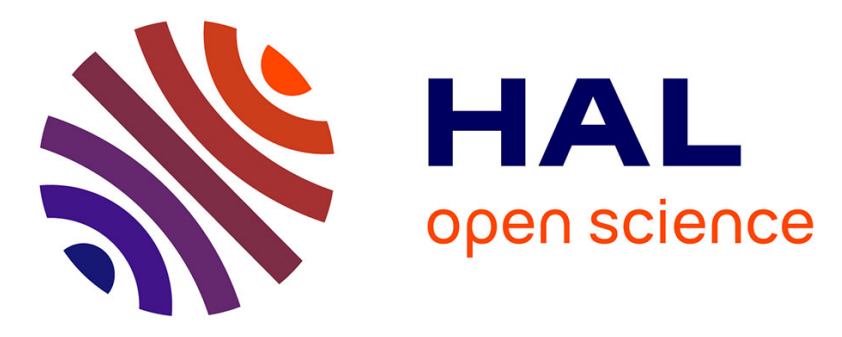

\title{
Microscale viscosity imaging using heterodyne holographic analysis of nanorods rotation
}

Clémence Gentner, Robert Kuszelewicz, Pascal Berto, Jean-François Berret, Gilles Tessier

\section{- To cite this version:}

Clémence Gentner, Robert Kuszelewicz, Pascal Berto, Jean-François Berret, Gilles Tessier. Microscale viscosity imaging using heterodyne holographic analysis of nanorods rotation. Digital Holography and Three-Dimensional Imaging, Jul 2021, Washington, United States. pp.DW4C.6, 10.1364/DH.2021.DW4C.6 . hal-03453317

\section{HAL Id: hal-03453317 https://hal.science/hal-03453317}

Submitted on 28 Nov 2021

HAL is a multi-disciplinary open access archive for the deposit and dissemination of scientific research documents, whether they are published or not. The documents may come from teaching and research institutions in France or abroad, or from public or private research centers.
L'archive ouverte pluridisciplinaire HAL, est destinée au dépôt et à la diffusion de documents scientifiques de niveau recherche, publiés ou non, émanant des établissements d'enseignement et de recherche français ou étrangers, des laboratoires publics ou privés. 


\title{
Microscale viscosity imaging using heterodyne holographic analysis of nanorods rotation
}

\author{
Clémence Gentner, ${ }^{1}$ Robert Kuszelewicz, ${ }^{1}$ Pascal Berto, ${ }^{1,2}$ Jean-François Berret, ${ }^{3}$ \\ and Gilles Tessier ${ }^{1, *}$ \\ ${ }^{1}$ Photonics Department, Institut de la Vision, UMR 7210 CNRS, INSERM, Sorbonne Université, 17 Rue Moreau, \\ F-75012 Paris, France \\ ${ }^{2}$ Université Paris Descartes, 45 rue des Saints-Pères, 75006 Paris, France \\ ${ }^{3}$ Matière et Systèmes Complexes, UMR 7057 CNRS, Université Denis Diderot Paris-VII, Bâtiment Condorcet, 10 \\ rue Alice Domon et Léonie Duquet, 75205 Paris, France \\ *gilles.tessier@inserm.fr
}

\begin{abstract}
By measuring the blinking induced by nanorods either in active (magnetically forced) or passive (Brownian) rotation with heterodyne holography, we performed high resolution viscosity imaging, an essential tool to study many microfluidic and biological processes. (C) 2021 The Author(s)
\end{abstract}

\section{Introduction}

Many processes in microfluidics and biology are driven or affected by local viscosity. At small scales, viscosity and other mechanical properties cannot be probed with macroscopic instruments, and their measurement defines a broad research field called microrheology [1]. Global viscosity measurements, particularly in cells, can be achieved by several methods including AFM, surface measurement of acoustic waves, biomembrane force probe, micropipette aspiration or Dynamic Light Scattering (DLS). Local viscosity measurements inside a cell are conducted via in-cell transducers either in active [2] or passive [3] motion. Fluorescence lifetime microscopy (FLIM) on genetically modified molecular rotors has recently shown its ability to image viscosity [4, 5]. However, photo-bleaching issues prohibit any experiment exceeding a few minutes, which is obviously problematic to study long-lasting phenomena. Here, we propose an optical method based on heterodyne holography to study the dynamics of nanorod rotation.

\section{Heterodyne holography of nanorods}

\subsection{Nanorods rotation detection}

The relatively large scattering cross-section of metallic or plasmonic nanoparticles offers a way to reduce probe size. By adjusting the concentration to fill the cell volume without inducing interaction between nanoparticles, high imaging resolution can be reached, and can even be pushed beyond the diffraction limit using 3D superlocalization [6].

To be able to optically detect the rotation, ellipsoidal nanoparticles (nanorods) are used: the alignment between their long axis and the illumination polarisation corresponds to a scattering maximum, whereas the orthogonal configuration corresponds to a minimum. Therefore, the blinking frequencies of an illuminated nanorod reveals its rotation dynamics, from which viscosity can be deduced.

\subsection{Heterodyne holography set-up}

The short exposure time of ultrafast cameras is not compatible with the detection of weak scatterers unless high illumination powers are used, which is impossible in living media. Heterodyning is a powerful alternative: by using a frequency beating at frequency $\Delta F$ (up to several $\mathrm{MHz}$ ), any variation occurring at $\Delta F$ in the sample can be imaged after demodulation on a dark background, even with a low frame rate, sensitive camera. The interferometric nature of holography is appropriate to set up such heterodyne detection, by introducing acoustooptical modulators (AOMs) in both the illumination and reference paths (see Fig.1) [7-9].

\subsection{Viscosity imaging via Brownian rotation measurement}

Using the set-up shown in Fig.1, we imaged the Brownian rotation of gold nanorods over a broad frequency range $(0-10 \mathrm{MHz})$ (Fig.1(a)). While a single measurement at any frequency results in a qualitative viscosity contrast image, a frequency scan offers quantitative viscosity values through the relation between the fit parameter $\gamma$ of the 
spectrum and the viscosity $\eta$ [3]. An image of two media of viscosities 0.9 and $1.3 \mathrm{mPa} . \mathrm{s}$ respectively is shown in Fig.1(c,d) and demonstrates high resolution, high precision live viscosity imaging.

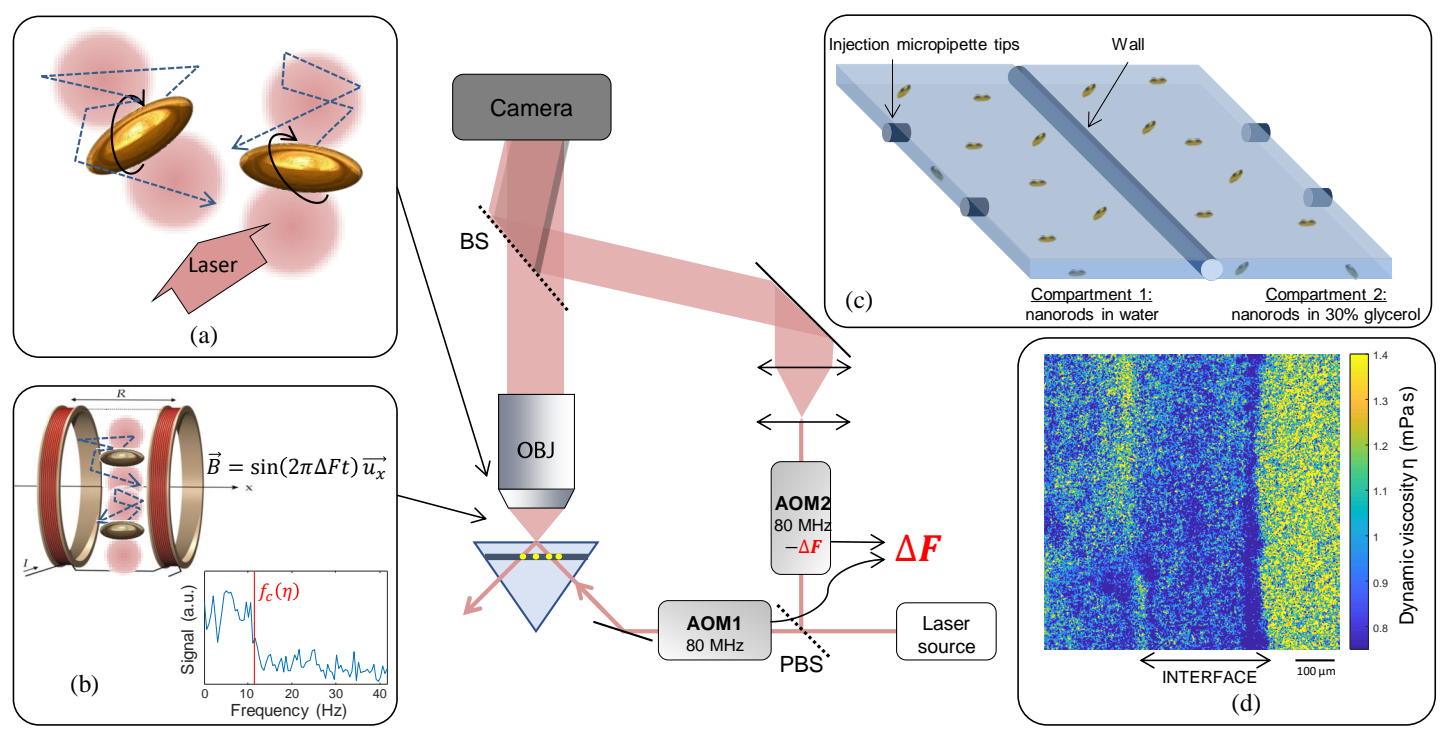

Fig. 1. Schematics of the heterodyne holography set-up. Light is coupled via a glass prism to obtain dark-field illumination. BS: beam splitter. (a): Scattering by gold nanorods in Brownian rotation. (b): Magnetic nanorods in an oscillating magnetic field. The heterodyne intensity spectrum of one nanorod while scanning the field's frequency displays a cutoff frequency that depends on viscosity. (c): Sample description: two solutions of different viscosities containing the same nanorod concentration have been injected in two microfluidic chambers separated by a thin $(300 \mu \mathrm{m})$ wall. (d): Viscosity image of (c). The average value in the left compartment is $0.91 \pm 0.02 \mathrm{mPa} . \mathrm{s}$ for a theoretical value $0.93 \pm 0.02 \mathrm{mPa} . \mathrm{s}$, and in the right compartment $1.21 \pm 0.02 \mathrm{mPa} . \mathrm{s}$ for a theoretical value $1.28 \pm 0.02 \mathrm{mPa} . \mathrm{s}$

\subsection{Magnetic nanorods rotation measurement}

Sub-micrometric iron oxide rods were synthesized [2] and their rotation spectra when submitted to a rotating magnetic field were recorded using the same method. The cut-frequency $f_{c}$ at which the rod rotation frequency decorrelates from that of the magnetic field is measured up to the $\mathrm{kHz}$ (Fig.1(b)), leading to the local viscosity $\eta$ through Eq.1 [2] where $\Delta \chi$ relates to the susceptibility anisotropy, $L$ and $D$ are respectively the length and the diameter of the rod, $H$ is the magnetic field strength, and $g\left(\frac{L}{D}\right)=\ln \frac{L}{D}-0.662+0.917 \frac{D}{L}-0.050\left(\frac{D}{L}\right)^{2}$.

$$
f_{c}=\frac{3 \pi}{4} \frac{\mu_{0} \Delta \chi}{\eta} g\left(\frac{L}{D}\right) \frac{D^{2}}{L^{2}} H^{2}
$$

\section{Conclusion}

Viscosity imaging with heterodyne holography of both Brownian and magnetic nanorods rotation is demonstrated. Applications in live cells are ongoing and promise high resolution 3D live imaging of viscosity during transformations like meiosis in oocytes.

\section{References}

1. F. Ortega, H. Ritacco, R. G. Rubio, Current Opinion in Colloid and Interface Science 15, 237-245 (2010).

2. J. F. Berret, Nature Communications 7 (2016).

3. C. Gentner, R. Kuszelewicz, P. Berto, H. Khelfa, G. Tessier, Optics Express 29, 527 (2021).

4. M. K. Kuimova, Physical Chemistry Chemical Physics 14, 12651-13102 (2012).

5. J. E. Chambers et al., ACS Nano 12, 4398-4407 (2018).

6. P. Bon et al., Nature Communications 6 (2015).

7. E. Absil et al., Optics Express 18, 780 (2010).

8. S. Y. Suck, S. Collin, N. Bardou, Y. De Wilde, G. Tessier, Optics Letters 36, 849 (2011).

9. M. Atlan et al., Optics Letters 33, 500 (2008). 